\title{
Knowing where is different from knowing what: Distinct response time profiles and accuracy effects for target location, orientation, and color probability
}

\author{
Syaheed B. Jabar ${ }^{1}$ • Alex Filipowicz ${ }^{2}$ • Britt Anderson ${ }^{1,3}$
}

Published online: 25 August 2017

(C) The Psychonomic Society, Inc. 2017

\begin{abstract}
When a location is cued, targets appearing at that location are detected more quickly. When a target feature is cued, targets bearing that feature are detected more quickly. These attentional cueing effects are only superficially similar. More detailed analyses find distinct temporal and accuracy profiles for the two different types of cues. This pattern parallels work with probability manipulations, where both feature and spatial probability are known to affect detection accuracy and reaction times. However, little has been done by way of comparing these effects. Are probability manipulations on space and features distinct? In a series of five experiments, we systematically varied spatial probability and feature probability along two dimensions (orientation or color). In addition, we decomposed response times into initiation and movement components. Targets appearing at the probable location were reported more quickly and more accurately regardless of whether the report was based on orientation or color. On the other hand, when either color probability or orientation probability was manipulated, response time and accuracy improvements were specific for that probable feature dimension. Decomposition of the response time benefits demonstrated that spatial probability only affected initiation times, whereas manipulations of feature probability affected both initiation and movement times. As detection was made more difficult, the two effects further
\end{abstract}

Britt Anderson

britt.anderson@uwaterloo.ca

1 Department of Psychology, University of Waterloo, 200 University Avenue West, Waterloo, ON N2L 3G1, Canada

2 Department of Neuroscience, University of Pennsylvania, Philadelphia, PA, USA

3 Centre for Theoretical Neuroscience, University of Waterloo, Waterloo, ON, Canada diverged, with spatial probability disproportionally affecting initiation times and feature probability disproportionately affecting accuracy. In conclusion, all manipulations of probability, whether spatial or featural, affect detection. However, only feature probability affects perceptual precision, and precision effects are specific to the probable attribute.

Keywords Perceptual learning · Attention: space-based . Attention: object-based

\section{Significance statement}

We are sensitive to environmental information. Objects that preferentially appear in certain locations or that contain particular features (e.g. colors) are perceived better. Attention research suggests that 'attending' to space differs from 'attending' to features. We examined how spatial learning differs from feature learning. While spatial probability effects manifested in all scenarios, feature probability effects only manifested when that feature was to be discriminated, and in these situations aided perceptual discrimination to a greater extent than spatial probability. These results suggest that although we are sensitive to both spatial and feature information, they affect our perceptual abilities in different ways.

\section{Introduction}

Probability affects perception. Frequently occurring objects are detected more quickly and more accurately than infrequently occurring ones (Hon, Yap, \& Jabar, 2013; Laberge \& Tweedy, 1964; Miller \& Pachella, 1973). Objects in probable locations are detected more quickly and consistently than objects at improbable locations are (Druker \& Anderson, 
2010; Fecteau, Korjoukov, \& Roelfsema, 2009; Geng \& Behrmann, 2005; Jiang, Sha, \& Remington, 2015; Rich et al., 2008; Vincent, 2011; Walthew \& Gilchrist, 2006; Wolfe et al., 2007 ).

Although they might seem similar on a surface level, different forms of probability manipulations can create distinct effects. In Jabar and Anderson (2017b), we used a perceptual estimation task and found that orientation probability increased the precision of an orientation estimation, which did not occur with spatial probability. Instead, spatial probability improved the ability to perceive the gratings detected as a decrease in guessing events, but it did not change the precision of orientation reports for the perceived proportion of trials. The objective of the present set of experiments, motivated by this prior study, is to further explore the similarities and differences between spatial and feature probability. Particularly, we were motivated by two questions: (1) Do these effects manifest under the same situations? (2) When they do manifest, do they affect responses the same way?

\section{Feature-specific versus domain-general effects}

We have previously argued that orientation probability is likely to be specific in improving the perception of orientations (Anderson, 2014; Jabar \& Anderson, 2015) as opposed to other features shared by the target, because it results in an experience-dependant sharpening of the responses of V1 orientation-selective neurons, akin to what occurs with orientation training in monkeys (Ringach, Hawken, \& Shapley, 1997; Schoups, Vogels, Qian, \& Orban, 2001). More direct evidence of this experience-dependent tuning comes from our finding that orientation probability reduces the amplitude of the electrophysiological C1 component (Jabar, Filipowicz, \& Anderson, 2017), which is thought to index early V1 activity (Di Russo, Martínez, Sereno, Pitzalis, \& Hillyard, 2002). An alternative argument could be made regarding feature probability as a form of feature-based attention, which has also been suggested to result in neural tuning (Carrasco, 2011; Çukur, Nishimoto, Huth, \& Gallant, 2013; David, Hayden, Mazer, \& Gallant, 2008; Ling, Jehee, \& Pestilli, 2015; Paltoglou \& Neri, 2012). Both accounts, which rely on domain-specific tuning mechanisms, lead to the conclusion that feature probability effects should be specific to the feature whose probability is being manipulated.

In contrast, space-based manipulations are thought to be more related to gain mechanisms (Carrasco, 2011), such as increasing the input baseline of neural responses (Cutrone, Heeger, \& Carrasco, 2014). If these baseline increases occur for all neurons coding for that space, it might account for how spatial probability has general effects. Spatial probability helps in locating an object: Jiang et al. (2015) used a T-among-L search display with the target appearing 3 times more likely in one particular quadrant. The targets in the probable location were detected significantly faster. Spatial probability also facilitates nonspatial dimensions, such as improving color detection (Druker \& Anderson, 2010), speeding up orientation judgments (Jabar \& Anderson, 2017b), and improving sequence learning (Filipowicz, Anderson, \& Danckert, 2014).

If feature probability results in feature-specific tuning changes while spatial probability results in general baseline changes, then differences across these manipulations should generalize beyond estimation tasks. For example, in a two-alternative forced-choice (2AFC) task, participants can be asked to report whether a target stimulus has a left-titling ( () or right-tilting (/) orientation. If spatial probability results in domain-global effects, it should facilitate discrimination of orientations. However, because orientation probability also improves perceptual precision where spatial probability does not (Jabar \& Anderson, 2017b), orientation probability should show bigger effects on discrimination accuracy than on spatial probability, especially when the discrimination task is perceptually difficult. In addition, if feature probability only results in feature-specific effects, one might expect that while manipulations of orientation probability might aid in orientation discrimination, manipulations of color probability should not. If this specificity versus generality hypothesis is true, the reverse situation must also hold: While both spatial and color probability should aid in color discrimination, orientation probability should not.

\section{Initiation versus movement times}

While 2AFC tasks can provide much information about the effect of spatial/featural manipulations on perception across a series of trials (e.g. Cutrone et al., 2014; Ling, Liu, \& Carrasco, 2009), on any single trial it typically leads to binary data - either one or the other button is pushed. Such binary classifications can miss important details. Prior data from estimation tasks suggest that initiation times (ITs) are more closely linked to perceptual precision while movement times (MTs) are more closely linked to confidence (Jabar \& Anderson, 2015). However, standard keyboards only register a button press when the key travel reaches some threshold, and not when the key has started to move. Therefore, it would be impossible to distinguish how much time was required to initiate the button press from the time used in the actual motion of the button press. Decomposing of RTs into ITs and MTs can instead be achieved through the use of levers or triggers (e.g. Smeets, Wijdenes, \& Brenner, 2016), which can report the state of the response in a continuous fashion.

We implemented a novel method for $2 \mathrm{AFC}$ tasks that uses the triggers on an Xbox controller to enrich the measuring of the dynamics that goes into making a 'choice'. Each of the two options is tied to a separate trigger. IT can be taken as the period from the time of stimulus onset to the moment a trigger pull is initiated. MT is taken from the moment the initiation 
occurs to the time the trigger state reaches a predetermined threshold value. If both feature and spatial probability affect the time taken to perceive targets, both should affect ITs. MTs, on the other hand, might be more indicative of confidence than accuracy (Jabar \& Anderson, 2015). MT is dependant on other aspects of the response profile, such as the baseline level of preparation, the force of the response, and whether the participant vacillates between the two options. Prestimulus baseline levels of preparation should be constant if the probability of either response is kept the same. However, response force has been suggested to be affected by stimulus probability, even when response probability is controlled for (Mattes, Ulrich, \& Miller, 2002), suggesting that probability also has nonperceptual effects. By looking at which component or components of RTs are affected by our manipulations, we can have a better idea of the potential mechanisms involved.

In summary, our aim was to examine differences between feature and spatial probability across two dimensions. Do the two manipulations differ in how domain general they are? Do they have similar response profiles? We designed a series of modified 2AFC experiments that are procedurally similar to allow for comparisons. In Experiment 1-ori and Experiment 1-col, we looked at the effects of orientation and color probability when that feature whose probability was manipulated was relevant for the detection task, respectively. In Experiment 2-ori and Experiment 2-col, we performed identical probability manipulations, but the task now depended on detecting the feature whose probability was not manipulated. In each experiment there was also a block where spatial probability was manipulated. Despite always being 'irrelevant', spatial probability had a consistent effect on ITs and accuracy. In contrast, feature probability effects manifested only when the probable feature was relevant for the discrimination (Experiment 1), but not when irrelevant (Experiment 2). In addition, only feature probability affected MTs. Experiment 3 looked at orientation and spatial probability with a more difficult orientation discrimination task, with the result that orientation probability showed an increased effect on task accuracy.

\section{Experiment 1}

\section{Method}

\section{Participants}

For Experiment 1 -ori, 16 participants (median age $=21$ years) were recruited from the University of Waterloo (eight females, eight males), in exchange for course credits. All reported themselves right-handed. For Experiment 1-col, 18 additional participants (median age $=20$ years) were recruited (nine females, nine males). Seventeen reported themselves to be right-handed. All participants had normal or corrected-tonormal vision and were not colorblind. This study was approved by the university's Office of Research Ethics.

\section{Stimuli}

Oriented square-wave gratings with a circular mask were the target stimuli. These were shown at 50\% maximum contrast and colored either blue or green (see Fig. 1). These green and blue patches were isoluminant at $40 \mathrm{~cd} / \mathrm{mm}^{2}$, as measured by a ColorCAL MKII colorimeter. The gratings had a spatial frequency of four cycles per degree of visual angle, and were presented on a gray background with a similar luminance. When viewed from a distance of $60 \mathrm{~cm}$, the gratings subtended approximately four degrees of visual angle both vertically and horizontally. Targets were presented either above or below a black fixation cross, at an eccentricity of 4 degrees.

Only one grating was presented on each trial. These gratings always had a spatial attribute (top or bottom), a color attribute (green or blue), and an orientation attribute (right titling or left tilting). Both Experiment 1-ori and Experiment 1-col had a feature probability block and a spatial probability block. In the spatial block, gratings occurred in one of the two locations $80 \%$ of the time (counterbalanced across participants), and the features (color and orientation) was equiprobable for each location (see Fig. $1 \mathrm{~b}$ for sample sequences). The practice block had uniformly distributed elements (location, color, orientations).

For Experiment 1-ori, the feature block had equiprobable locations and color, but orientation probability was manipulated. Feature probability was manipulated in a locationcontingent manner. When the grating occurred in one location, it was $80 \%$ likely to be right tilting (/: 45 degrees). In the other location, the left tilt $(\backslash: 135$ degrees) was $80 \%$ likely. Note how the distribution for the feature block differs from that of the spatial block (see Fig. 1b). The location-orientation contingencies were counterbalanced across participants. The participants' task was to report the tilt. Key assignments were also counterbalanced across participants. Because of the locationcontingent probability mapping, the probability of response was always equal across the two triggers. This locationcontingent probability mapping also made the feature probability uniform if space is ignored.

For Experiment 1-col, the feature block had equiprobable locations and orientations, but color probability was manipulated, again in a location-contingent manner. When the grating occurred in one location, it was $80 \%$ likely to be blue. In the other location, green was $80 \%$ likely. This was counterbalanced across participants. Participants were told to detect color and were assigned one trigger for green and the other for blue. Key assignment was counterbalanced across participants. 


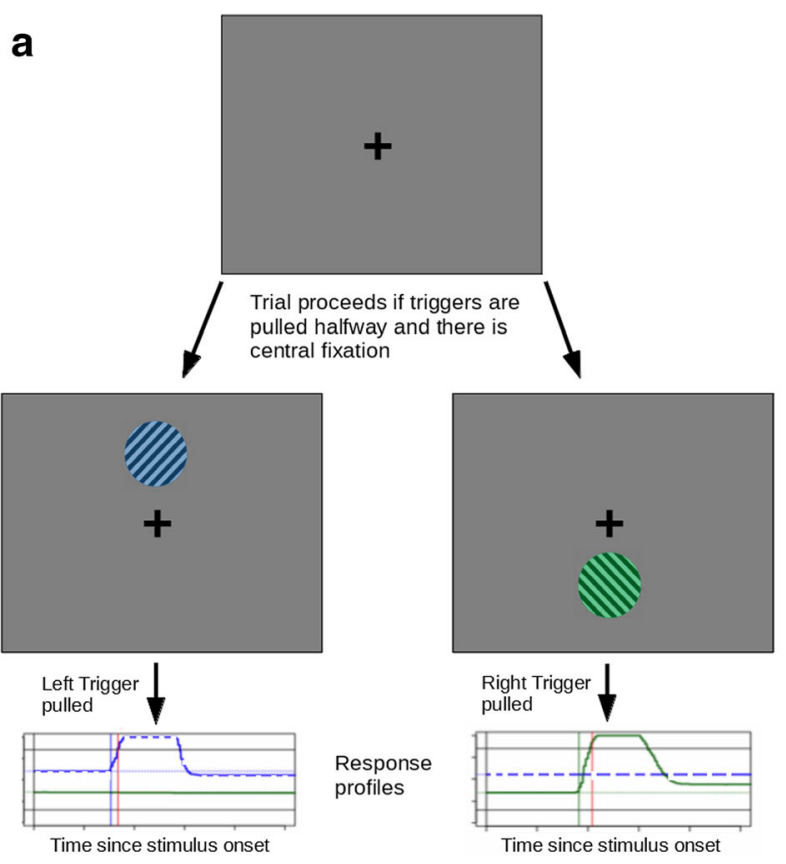

b

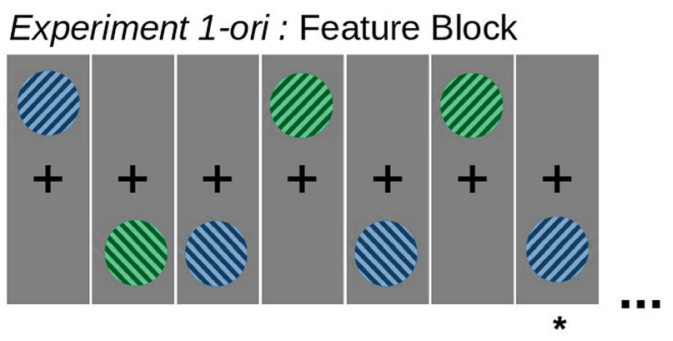

\section{Experiment 1-ori : Spatial Block}

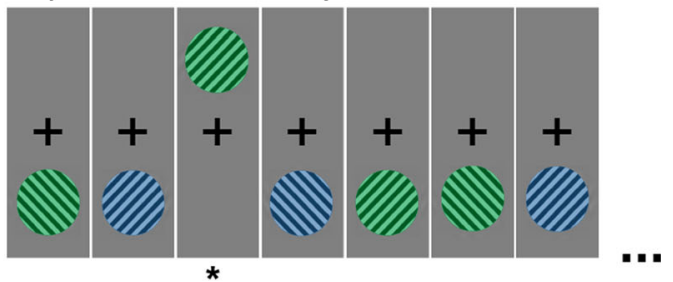

orientation with one trigger being assigned to each of 45 and 135 degrees. Note how the two triggers can be operated independently and simultaneously. b Trial distribution for Experiment 1-ori for the feature (orientation) and spatial block. Asterisks indicate a low-probability trial for that block. The probable orientation/space is counterbalanced across participants. (Color figure online)
Within a block, probability distributions were maintained (e.g. in the spatial block, for every set of 20 trials, there were 16 gratings on the top, four gratings the bottom, etc.). Participants were not informed about these probability distributions. While the probability type was changed across blocks, this was unannounced, and the task from a participant's view remains the same: In Experiment 1-col, the task was always to detect color; in Experiment 1-ori, the task was always to detect orientation. Auditory feedback was given after each response to maintain motivation. A high-pitched sound indicated a correct response. A lower pitch sound indicated an error.

\section{Equipment}

All experiments used the same equipment. Experiments were coded in Python and run on a computer using a Linux operating system. Stimuli were displayed on a gamma-corrected CRT monitor that refreshed at $89 \mathrm{~Hz}$ (mean refresh $=11.27 \mathrm{~ms}$, $S D=0.07 \mathrm{~ms}$ ). An Xbox controller was connected via USB and the xboxdrv package (https://aur.archlinux.org/packages/ xboxdrv/) allowed access to the controller for recording trigger force and excursion. Responses were made using the two back triggers of the Xbox 360 wired controller. Participants were instructed to hold the triggers half-depressed with their index fingers. This allowed us to detect small changes in force at baseline or preceding responses. A continuous measure of trigger depression (recorded at $2000 \mathrm{~Hz}$ ) was obtained for each trigger. The output scaled from -1 (no pressure) to +1 (fully depressed). A value of +0.7 was fixed as the detection threshold, and a value of -0.7 or less caused the motors in the handles of the controller to rumble, giving the participant haptic feedback, indicating that they should increase their pressure on the trigger. Stimuli were only displayed when both triggers values were within the -0.7 to +0.7 range (i.e. the next trial began only after the participant relaxed their response from the previous trial). See Fig. 2 for example response profiles.

Eye tracking used an Eyelink 1000, recording the dominant eye at $2000 \mathrm{~Hz}$, and using both pupil and corneal signals. Participant head position was stabilized using a chin and forehead rest. Drift corrections were done every 100 trials (approximately every $2-4$ minutes). A recalibration was done if the fixation was lost. Recalibrations were also done in between blocks (at approximately the 12-minute mark). The next trial began only when the participant fixated on the fixation cross, within a radius threshold of 0.5 degrees visual angle. This was set up so that stimuli in the same on-screen locations were presented at roughly the same peripheral location retinotopically. This also prevented anticipatory saccades prior to a trial.

\section{Procedure}

Prior to the task, participants were instructed as to the trigger assignment and to maintain pressure at about a halfway point 


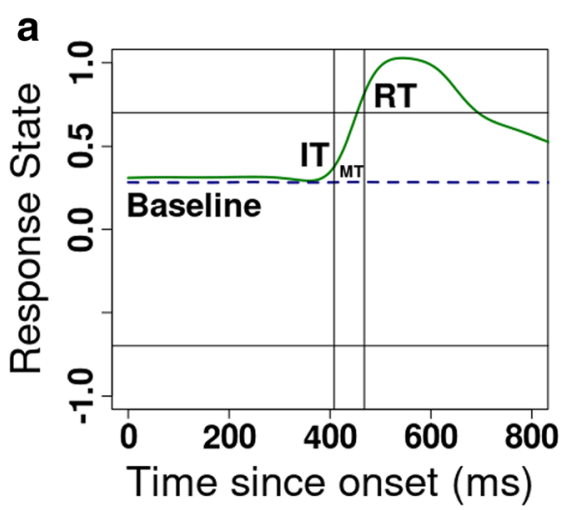

Fig. 2 Trigger profiles of actual sample trials from one participant. a Typical trial with only one response. b Trial with a vacillation in responses that was too late. $\mathbf{c}$ Trial with a vacillation in responses that was in time. The two lines indicate the two possible response options (left trigger $=$ blue dotted line , right trigger = green solid line $)$. The upper

unless making a response, in which case they were to pull the appropriate trigger as fast as possible. Regardless of whether participants were in the practice, spatial, or feature block, the task was always the same: Either report orientation in Experiment 1-ori or color in Experiment 1-col. Participants were not informed of the probability manipulations.

The experiment began with 20 practice trials. The next 800 trials were split into two blocks. The first block was either the spatial or feature block, and after a short break, the other block type was administered. Trials began with a fixation phase, where only the black fixation cross was shown. If there was central fixation ( 0.5 degree radius) and the appropriate trigger levels (between -0.7 and +0.7 ) were detected, the grating appeared between $250 \mathrm{~ms}$ and $500 \mathrm{~ms}$ (uniform distribution) later. The stimulus remained on-screen until a response was made, at which point auditory feedback was given, and the stimulus disappeared (after $500 \mathrm{~ms}$ ). Participants were asked to minimize blinking when the gratings were on the screen, preferably blinking only during the feedback period.

During the drift correction (every 100 trials), participants were told to let go of the controller and to relax their hands and blink a few times. This was to minimize fatigue. During the break (after 400 trials), participants were free to remove their head from the chin rest. The experiment lasted approximately 20-25 minutes, after which a questionnaire was administered.

\section{Postexperiment questionnaire}

Before debriefing, participants were given a short questionnaire consisting of the following six open-ended questions. This questionnaire was modified from the one used in Jabar and Anderson (2017b) to include a question on color (Question 5).

1. Did anything about the experimental task stand out to you?

2. Please describe any strategies you may have used.

\section{b}
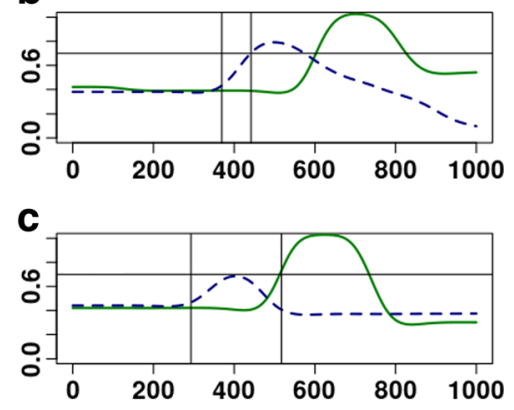

black line indicate the detection threshold, the bottom black line indicates the pressure threshold (the controller rumbles below this value). Note how the time required to cross the detection threshold (RT) might be affected by the vacillations. The two vertical lines represent the initiation time and RT, respectively. (Color figure online)

3. Did you feel that you perceived some stimuli better or differently than others, or in certain cases? Did you notice any change over time in your experience?

4. Do you think that some orientations are more likely at certain times? If yes, please elaborate.

5. Do you think that some colors are more likely at certain times? If yes, please elaborate.

6. Do you think that some locations are more likely at certain times? If yes, please elaborate.

\section{Analysis}

Analysis was done using the $\mathrm{R}$ statistical software package ( $\mathrm{R}$ Core Team, 2016). With the exception of comparisons across experiments, all statistical tests were done within subjects. Preprocessing was done by running a smoothing spline (see Fig. 2a). Response baselines were taken as the median trigger value between $50 \mathrm{~ms}$ prior and after stimulus onset. Velocity profiles were taken as a first derivative, and acceleration as the second. Trigger acceleration was taken as an indication of force, since Force $=$ Mass $\times$ Acceleration, and mass can be assumed constant (the units of the force/acceleration is therefore in trigger distance per millisecond squared). Vacillations were identified from the points where acceleration goes from positive to zero to negative (the point where the trigger is released). A trial could have multiple initiations, either within or across triggers. Initiation times (ITs) were back-calculated from these vacillations/turning points (e.g. where there is a +0.05 increase over the baseline within $100 \mathrm{~ms}$ prior to the turning point). Reaction times (RTs) were taken as when a trigger crossed the +0.7 threshold. Note that participants could have tried to correct their responses either unsuccessfully (see Fig. 2b) or successfully (see Fig. 2c). Number of initiations takes into account these 'unsuccessful corrections'. Movement times (MTs) were taken as the difference between 
the RT and the first IT. Bayesian inference testing was conducted using the BayesFactor R package (Morey, Rouder, \& Jamil, 2015), where the measure of interest is the Bayes factor (BF).

Accuracy measures were taken at two points. Initial accuracy was taken at the point of first initiation (whether or not the participant started by pulling the correct trigger). Final accuracy was taken at the point one of the triggers crossed the threshold, where participants were given feedback on their response. Change in accuracy due to vacillations was taken as a difference between these two points.

\section{Results}

Response baselines $(M=0.0, S D=0.3)$, as per instructions, were halfway between a complete pull $(+1)$ and a complete release $(-1)$, with some variability across participants. Importantly, these baselines were equivalent across all blocks of all experiments (all $p \mathrm{~s}>.05$ ). This was likely because in all cases, the probability of the left and right response was 50/50, suggesting that there was no prestimulus response preparation. It is conceivable that participants could have been inclined to respond with the probable trigger once the stimulus has been shown. To rule out a poststimulus response-preparation account, for each trial, the trigger states just $(10 \mathrm{~ms})$ prior to the point of response initiation were also noted and compared against the response baselines for that trial. For both the initiated and noninitiated triggers, and in all blocks of all the experiments, there was no change in response states between the time of stimulus presentation and the time of initiation (all $p \mathrm{~s}$ $>.05$ ).

There was also no clear bias towards the left or right trigger ( $p$ s >.05), despite most participants being right-handed. Handedness is unlikely to affect the results because all stimuli-to-response associations were counterbalanced across participants. Potential order effects (e.g. whether the spatial or feature block was first) were examined, and the data were found to be independent of order $(p s>.05)$.

\section{Experiment 1-ori (spatial + orientation probability, orientation detection)}

Reaction time For Experiment 1-ori, in the block where spatial probability was unequal there was an effect of spatial probability on RT (see Fig. 3). Orientations at the highprobability location were reported faster $(M=564 \mathrm{~ms}, S D=$ $96 \mathrm{~ms})$ than at the low-probability location $(M=631, \mathrm{~ms}, S D$ $=112 \mathrm{~ms}), t(15)=9.45, p<.001$. Breaking down RT into the IT and MT components revealed that ITs were significantly affected by spatial probability, with orientations in the highprobability location being initiated faster $(M=451 \mathrm{~ms}, S D=$ $86 \mathrm{~ms})$ than at the low-probability location $(M=510 \mathrm{~ms}, S D=$ $88 \mathrm{~ms}), t(15)=10.07, p<.001$. However, there was no effect

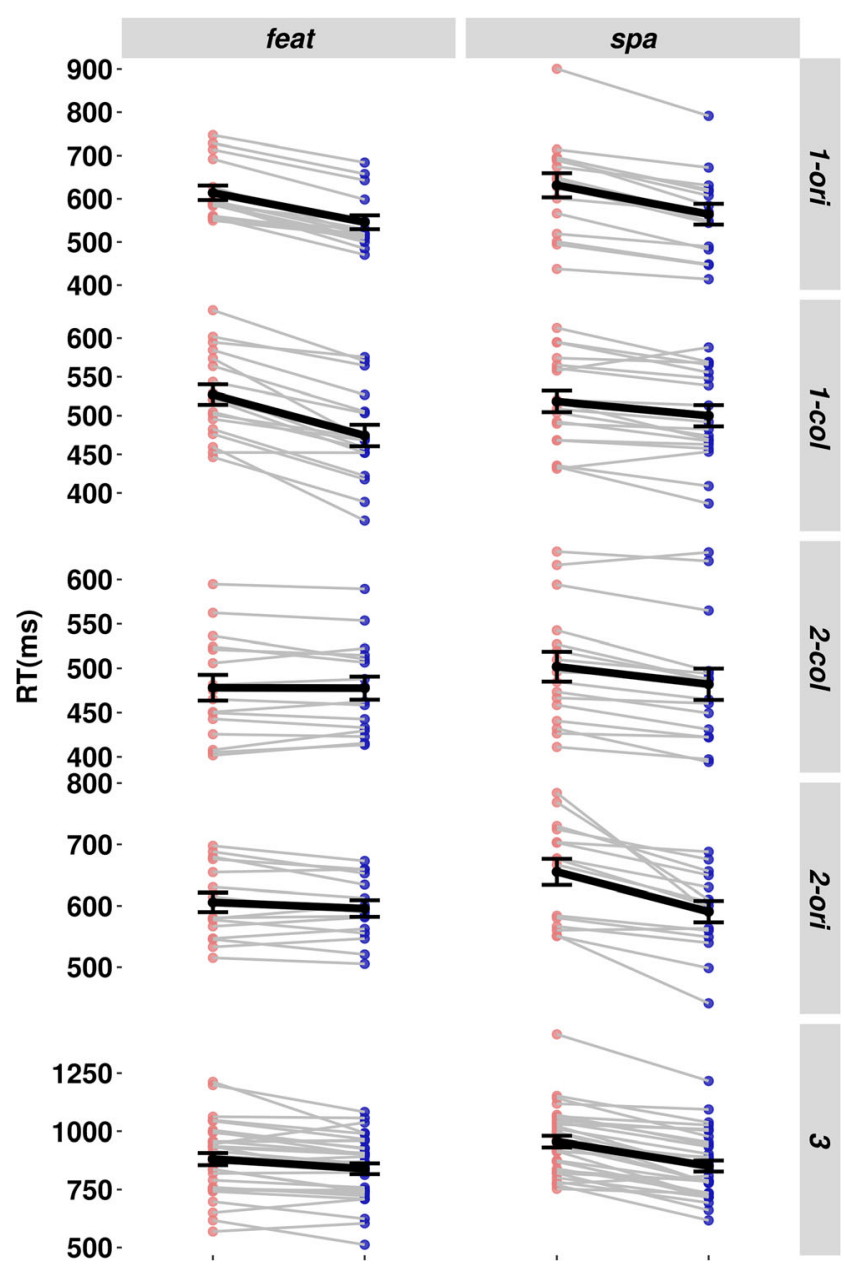

Fig. 3 Mean reaction time, and trends for each participant. Blue (dark) = high-probability; red (light) = low-probability. Panel rows refer to a different experiment, panel columns refer to either the spatial or feature block within the experiment. (Color figure online)

of spatial probability on MTs. MTs at high-probability locations ( $M=114 \mathrm{~ms}, S D=55 \mathrm{~ms}$ ) were not significantly different from the low-probability location $(M=121 \mathrm{~ms}, S D=63$ $\mathrm{ms}), t(15)=1.48, p=.160$. Force of the trigger pull was not affected across high $(M=0.002)$ and low $(M=0.002)$ probability locations, $t(15)=1.62, p=.126$.

For the feature (orientation) probability block there was also an effect of probability on RT. High-probability orientations were detected faster $(M=546 \mathrm{~ms}, S D=64 \mathrm{~ms})$ than the low-probability orientations were ( $M=614 \mathrm{~ms}, S D=67 \mathrm{~ms})$, $t(15)=10.93, p<.001$. ITs were significantly affected by feature probability, with responses to high-probability orientations being initiated faster ( $M=438 \mathrm{~ms}, S D=74 \mathrm{~ms}$ ) than to low-probability orientations ( $M=485 \mathrm{~ms}, S D=84 \mathrm{~ms}$ ), $t(15)$ $=7.55, p<.001$. Unlike with spatial probability, there was a significant effect of feature probability on MTs. MTs associated with high-probability orientations $(M=108 \mathrm{~ms}, S D=47$ $\mathrm{ms}$ ) were significantly faster than for low-probability orientations $(M=129 \mathrm{~ms}, S D=56 \mathrm{~ms}), t(15)=3.58, p=.003$. Force 
of the trigger pull was not affected across high $(M=0.003)$ and low $(M=0.002)$ probability orientations, $t(15)=1.38, p=$ .188 .

For comparison, the effect of spatial probability on RT ( $M$ $=67 \mathrm{~ms}, S D=28 \mathrm{~ms}$ ) and the effect of feature probability on RT $(M=68 \mathrm{~ms}, S D=25 \mathrm{~ms})$ were not significantly different from one another, $t(15)=0.17, p=.870$. Bayesian testing $(\mathrm{BF}$ $=.259[ \pm<0.01 \%])$ supports the null hypothesis: The effect of spatial and feature probability on total RT was equivalent, even though the effect on the IT and MT components differed.

Accuracy and vacillations For Experiment 1-ori, in the spatial block there was an effect of spatial probability on final accuracy (see Fig. 4), with orientations in the highprobability locations being responded to more accurately $(M=96.7 \%, S D=2.3 \%)$ than in the low-probability locations $(M=95.0 \%, S D=2.9 \%), t(15)=3.37, p=.004$. There was no effect of spatial probability on the amount of vacillations, with orientations in the high-probability locations being as directly responded to $(M=0.174, S D=0.250)$ as in the low-probability locations $(M=0.174, S D=0.241)$, $t(15)=0.02, p=.987$. Bayesian testing $(\mathrm{BF}=.255[ \pm$ $<0.01 \%$ ]) supports the null hypothesis. Vacillations improved initial to final accuracy $(p s<.05)$; this improvement was marginally different across high $(M=4.4 \%)$ and low $(M=5.1 \%)$ probability locations, $t(15)=1.96, p=.069$. Bayesian testing $(\mathrm{BF}=1.17[ \pm 0.01 \%])$ suggests that this trend is of minimal impact.

In the feature block, there was an effect of orientation probability on final accuracy, with high-probability orientations being responded to more accurately $(M=97.7 \%, S D=$ $2.0 \%)$ than the low-probability orientations $(M=89.3 \%, S D$ $=6.6 \%), t(15)=6.08, p<.001$. There was no significant effect of orientation probability on the amount of vacillations, with high-probability orientations being responded to more directly $(M=0.169, S D=0.263)$ than the low-probability orientations $(M=0.199, S D=0.260), t(15)=1.74, p=.102$. Bayesian testing $(\mathrm{BF}=0.875[ \pm 0.01 \%])$ supports the null hypothesis. Again, vacillations improved initial to final accuracy ( $p$ s $<$ $.05)$; this improvement was marginally different across high $(M=4.0 \%)$ and low $(M=7.0 \%)$ probability orientations, $t(15)=2.06, p=.057$. Bayesian testing $(\mathrm{BF}=$ $1.36[ \pm 0.01 \%])$ suggests that this trend is of minimal impact.

For comparison, the effect of spatial probability on final accuracy $(M=1.7 \%, S D=2.0 \%)$ was significantly smaller than the effect of orientation probability on final accuracy $(M$ $=8.4 \%, S D=5.5 \%), t(15)=4.94, p<.001$. The increase in accuracy due to vacillations was lower in the spatial probability block $(M=0.7 \%)$ than in the orientation probability block $(M=3.1 \%)$, although this was statistically insignificant, $t(15)$ $=1.70, p=.110$.

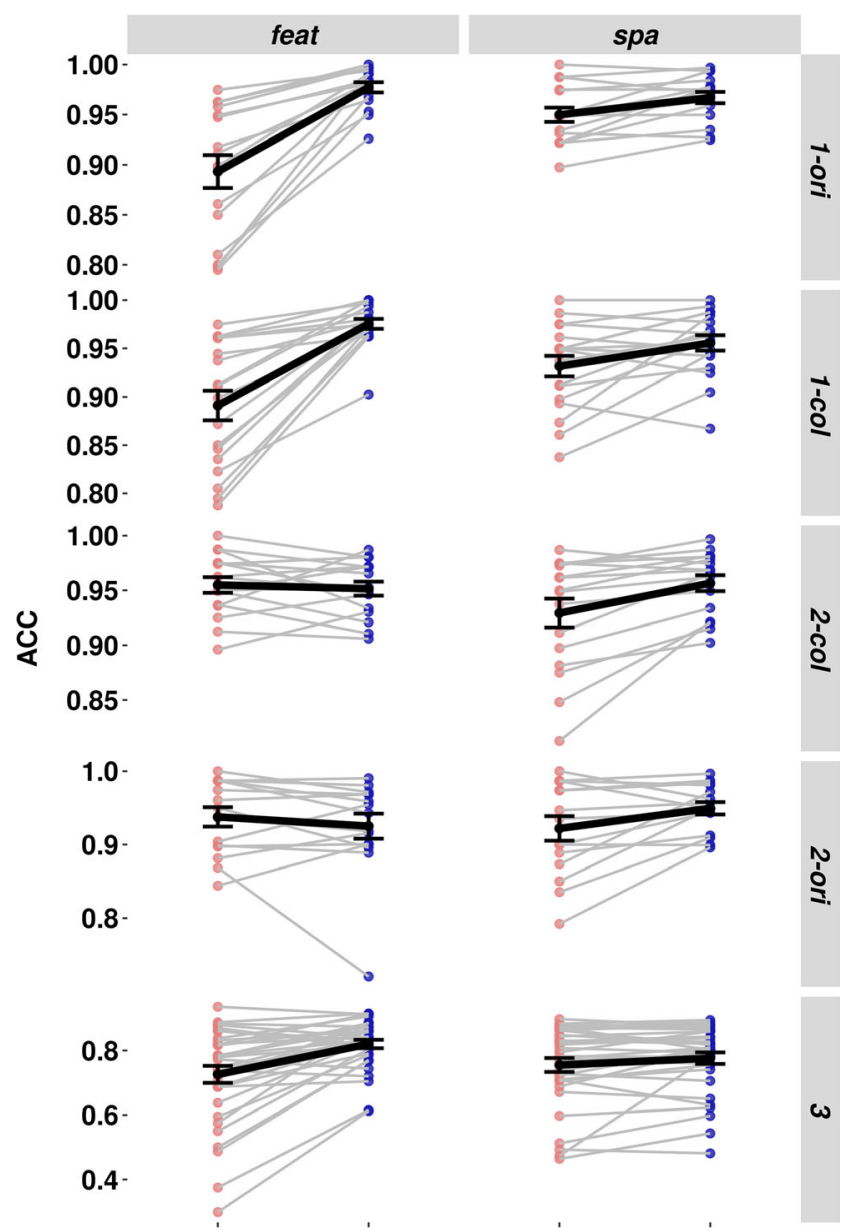

Fig. 4 Mean accuracy, and trends for each participant. Blue (dark) = high-probability; red (light) = low-probability. Panel rows refer to a different experiment, panel columns refer to either the spatial or feature block within the experiment. (Color figure online)

Experiment 1-col (spatial + color probability, color detection)

Reaction time For Experiment 1-col, in the spatial block there was an effect of spatial probability on RT, with colors at the high-probability location being detected faster $(M=500 \mathrm{~ms}$, $S D=58 \mathrm{~ms})$ than at the low-probability location $(M=518 \mathrm{~ms}$, $S D=58 \mathrm{~ms}), t(17)=3.63, p=.002$. ITs were significantly affected by spatial probability, with responses to colors in the high-probability locations being initiated faster $(M=400 \mathrm{~ms}$, $S D=68 \mathrm{~ms})$ than at the low-probability locations $(M=414$ $\mathrm{ms}, S D=77 \mathrm{~ms}), t(17)=2.87, p=.011$. However, there was no effect of spatial probability on MTs, with MTs associated with high-probability locations ( $M=99 \mathrm{~ms}, S D=26 \mathrm{~ms}$ ) not significantly different from low-probability locations $(M=$ $104 \mathrm{~ms}, S D=33 \mathrm{~ms}), t(15)=1.06, p=.304$. Force of the trigger pull was not affected across high $(M=0.002)$ and low $(M=0.001)$ probability locations, $t(17)=1.02, p=.321$.

For the feature (color) probability block, there was an effect of probability on RT, with high-probability colors being detected faster $(M=474 \mathrm{~ms}, S D=59 \mathrm{~ms})$ than low-probability 
colors $(M=527 \mathrm{~ms}, S D=56 \mathrm{~ms}), t(17)=8.89, p<.001$. ITs were significantly affected by color probability, with response to high-probability colors being initiated faster $(M=380 \mathrm{~ms}$, $S D=66 \mathrm{~ms})$ than low-probability colors $(M=416 \mathrm{~ms}, S D=$ $74 \mathrm{~ms}), t(17)=8.10, p<.001$. Unlike with spatial probability, there was a significant effect of color probability on MTs, with movement times associated with high-probability colors $(M=$ $95 \mathrm{~ms}, S D=25 \mathrm{~ms}$ ) being significantly faster than for lowprobability colors $(M=111 \mathrm{~ms}, S D=33 \mathrm{~ms}), t(17)=3.20, p=$ .005 . Force of the trigger pull was not affected across high $(M$ $=0.002)$ and low $(M=0.002)$ probability colors, $t(17)=0.54$, $p=.596$.

For comparison, the effect of spatial probability on RT ( $M$ $=18 \mathrm{~ms}, S D=21 \mathrm{~ms}$ ) and the effect of color probability on RT ( $M=52 \mathrm{~ms}, S D=25 \mathrm{~ms}$ ) were significantly different from one another, $t(17)=4.48, p<.001$. Across the two experiments, it was also evident that discriminating of orientations (Experiment 1-ori) was associated with a larger overall RT than the discrimination of colors (Experiment 1-col), for the spatial and feature blocks (both $p \mathrm{~s}<.001$ ).

Accuracy and vacillations For Experiment 1-col, in the spatial block there was an effect of spatial probability on final accuracy, with colors in the high-probability locations being responded to more accurately $(M=95.6 \%, S D=3.3 \%)$ than in the low-probability locations $(M=93.2 \%, S D=4.5 \%), t(17)=$ $2.60, p=.019$. There was no effect of spatial probability on the amount of vacillations, with colors in the high-probability locations being as directly responded to $(M=0.143, S D=$ 0.147 , as in the low-probability locations $(M=0.155, S D=$ $0.188), t(17)=0.83, p=.420$. Bayesian testing $(\mathrm{BF}=.328[ \pm$ $0.01 \%]$ ) supports the null hypothesis. Vacillations improved initial to final accuracy $(p \mathrm{~s}<.05)$; this improvement was marginally different across high $(M=4.8 \%)$ and low $(M=5.4 \%)$ probability locations, $t(17)=0.80, p=.434$. Bayesian testing $(\mathrm{BF}=0.323[ \pm 0.01 \%])$ supports the null hypothesis.

In the feature block, there was an effect of color probability on final accuracy, with high-probability colors being responded to more accurately $(M=97.5 \%, S D=2.2 \%)$ than in the low-probability colors $(M=89.1 \%, S D=6.5 \%), t(17)=$ $6.23, p<.001$. There was a marginally significant effect of color probability on the amount of vacillations, with highprobability colors being responded to more directly $(M=$ $0.143, S D=0.174)$ than the low-probability colors $(M=$ $0.177, S D=0.160), t(17)=2.04, p=.057$. Bayesian testing $(\mathrm{BF}=1.31[ \pm<0.01 \%])$ suggests that this trend is of minimal impact. Again, vacillations improved initial to final accuracy ( $p \mathrm{~s}<.05)$, and this improvement was smaller for the high $(M=$ $4.0 \%)$ than for low $(M=8.3 \%)$ probability colors, $t(17)=$ $4.48, p<.001$. Bayesian testing $(\mathrm{BF}=98.5[ \pm 0.01 \%])$ suggests that this trend is of moderate to strong impact.

For comparison, the effect of spatial probability on final accuracy $(M=2.4 \%, S D=3.9 \%)$ was significantly smaller than the effect of color probability on final accuracy $(M=$ $8.4 \%, S D=5.7 \%), t(17)=4.21, p<.001$. The increase in accuracy after vacillations was also lower in the spatialprobability block $(M=0.5 \%)$ than in the color-probability block $(2.9 \%), t(17)=3.05, p=.007$. Across the two experiments, discriminating of orientations (Experiment 1-ori) was not overall significantly more or less accurate than the discrimination of colors (Experiment 1-col) for either the spatial or the feature blocks (both $p \mathrm{~s}>.05$ ).

Postexperiment questionnaires Consistent with our previous studies (Jabar \& Anderson, 2015, 2017a, b), most of the participants did not realize that there were probability manipulations. Of the ones that did report something about the probability of a stimulus (seven out of 34 participants), only the spatial probability manipulation was accurate (e.g. 'the top was more likely'). Comments on the orientation or color probability were limited to "things that look like "/" were more frequent' or 'blue felt more likely'. Neither of these were true, because the feature probability was spatially contingent in all cases: If '/' was more likely when the grating appeared at the top location, ' '' was more likely when the grating appeared at the bottom location. The same trends in the questionnaire responses were seen in the later experiments as well.

\section{Discussion}

As with most studies on probability, participants were faster and more accurate when responding to probable objects than to improbable ones. Orientation, color, and spatial probability appear to have affected RT in a similar manner if one ignores the breakdown into MTs and ITs. While both spatial and feature probability affected ITs, only the latter had an impact on MTs, perhaps indicating increased confidence in responses. This logically should be tied down to the number of vacillations made or to response force, but those measures are likely too insensitive given a scenario where accuracies are high: Participants tend to initiate the correct trigger in the current experiment (see Experiment 3 for comparison with a more difficult task).

In addition to MT, both forms of feature probability (orientation/color) also seem to have an exaggerated impact on discrimination accuracy as compared to spatial probability, by a factor of 3 or 4 . One possible reason is that space in this context is uninformative to the response, whereas learning of the feature probabilities is informative. The other possibility is that feature probability is helping to shape perceptual precision. We return to this issue in Experiment 3.

Spatial and feature probability were nonequivalent manipulations in Experiment 1. The feature was directly informative to the response choice, but space was not. What happens when an irrelevant feature is made probable? Is the color of a grating with a probable orientation better discriminated than the color 
of a grating with an improbable orientation? Likewise, is the orientation of a grating with a probable color better detected than the orientation of a grating with an improbable color? A feature-tuning hypothesis might predict that only tuning the relevant feature is going to result in an effect. On the other hand, an object-based attentional hypothesis (e.g. Egly, Driver, \& Rafal, 1994) might predict that instances of objects with the probable feature might be attended to more than if the feature is improbable.

Experiment 2 examines this idea. By looking at irrelevant feature probabilities and comparing the results to Experiment 1 , we can determine what the impact of feature relevance (if any) might be on the probability effect, and to address whether feature probability is as domain general as spatial probability appears to be.

\section{Experiment 2}

\section{Method}

Experiment 2 is largely similar to Experiment 1 . As before, both experiments consisted of a spatial block and a feature block, not necessarily in that order. However, for the feature blocks, the discrimination task was on a feature that was not the feature that had its probability manipulated. Experiment 2col examined the orientation probability manipulation from Experiment 1-ori, but with the color-detection task of Experiment 1-col. Experiment 2-ori examined the colorprobability manipulation from Experiment 1-col, but with the orientation-detection task of Experiment 1-ori. The spatial blocks were identical.

\section{Participants}

For Experiment 2-col, 16 additional participants (median age $=21$ years) were recruited (11 females, five males), in exchange for course credits. All reported themselves to be right-handed. For Experiment 2-ori, 15 additional participants (median age $=21$ years) were recruited $(11$ females, four males). Fourteen reported themselves to be right-handed. Apart from flipping the task instructions, all aspects of Experiment 2 were equivalent to those of Experiment 1.

\section{Results}

Experiment 2-col (spatial + orientation probability, color detection)

Reaction time For Experiment 2-col, in the spatial block there was an effect of spatial probability on RT, with colors in the high-probability locations being detected faster $(M=482 \mathrm{~ms}$, $S D=71 \mathrm{~ms})$ than in the low-probability locations $(M=501$ $\mathrm{ms}, S D=67 \mathrm{~ms}), t(15)=5.14, p<.001$. ITs were significantly affected by spatial probability, with responses to colors in the high-probability locations being initiated faster $(M=392 \mathrm{~ms}$, $S D=71 \mathrm{~ms})$ than in the low-probability locations $(M=407$ $\mathrm{ms}, S D=71 \mathrm{~ms}), t(15)=4.61, p<.001$. However, there was no effect of spatial probability on MTs, with movement times associated with high-probability locations $(M=89 \mathrm{~ms}, S D=$ $31 \mathrm{~ms}$ ) not being significantly different from low-probability locations $(M=95 \mathrm{~ms}, S D=30 \mathrm{~ms}), t(15)=1.34, p=.201$. Force of the trigger pull was also not affected across highprobability $(M=0.002)$ and low-probability $(M=0.003)$ locations, $t(15)=0.97, p=.349$.

For the feature (orientation) probability block, there was no significant effect of probability on RT, with the color of highprobability orientations $(M=478 \mathrm{~ms}, S D=52 \mathrm{~ms})$ taking as long as the low-probability orientations $(M=478 \mathrm{~ms}, S D=58$ $\mathrm{ms}$ ) to detect, $t(15)=0.14, p=.887$. Unsurprisingly, neither IT nor MT nor force was affected by orientation probability (all $p s>.05)$. The Bayesian tests of orientation probability on RT in Experiment 1 -ori $(\mathrm{BF}=798183[ \pm<0.01 \%])$ and Experiment 2-col $(\mathrm{BF}=.258[ \pm<0.01 \%])$ clearly support opposing hypotheses.

Accuracy and vacillations For Experiment 2-col, in the spatial block there was an effect of spatial probability on final accuracy, with colors in the high-probability locations being responded to more accurately $(M=95.7 \%, S D=2.9 \%)$ than in the low-probability locations $(M=93.0 \%, S D=5.2 \%), t(15)=$ $3.57, p=.003$. There was no effect of spatial probability on the amount of vacillations, with colors in the high-probability locations being as directly responded to $(M=0.136, S D=$ $0.203)$ as in the low-probability locations $(M=0.153, S D=$ $0.214), t(15)=1.53, p=.148$. Vacillations improved initial to final accuracy $(p s<.05)$; this improvement was not significantly different across high-probability $(M=4.7 \%)$ and lowprobability $(M=5.2 \%)$ locations, $t(15)=.55, p=.589$.

In the feature block, there was no effect of orientation probability on final color accuracy, with colors of high-probability orientations being responded to as accurately $(M=95.2 \%, S D$ $=2.6 \%)$ as the low-probability orientations $(M=95.5 \%, S D=$ $2.9 \%), t(15)=0.49, p=.626$. As with RT, the Bayesian tests of orientation probability on color accuracy in Experiment 1-ori $(\mathrm{BF}=1141[ \pm<0.01 \%])$ and Experiment 2-col $(\mathrm{BF}=.284[ \pm$ $<0.01 \%]$ ) clearly support opposing hypotheses. There was no significant effect of orientation probability on the amount of vacillations or on the improvement of initial to final accuracy (all $p \mathrm{~s}>.05$ ).

Experiment 2-ori (spatial + color probability, orientation detection)

Reaction time For Experiment 2-ori, there was an effect of spatial probability on RT, with orientations in the high- 
probability locations being detected faster $(M=590 \mathrm{~ms}, S D=$ $67 \mathrm{~ms})$ than in the low-probability locations $(M=655 \mathrm{~ms}, S D$ $=82 \mathrm{~ms}), t(14)=4.70, p<.001$. ITs were significantly affected by spatial probability, with responses to orientations at the high-probability locations being initiated to faster $(M=479$ $\mathrm{ms}, S D=92 \mathrm{~ms})$ than at the low-probability locations $(M=$ $538 \mathrm{~ms}, S D=105 \mathrm{~ms}), t(14)=4.49, p<.001$. However, there was no effect of spatial probability on MTs, with movement times associated with high-probability locations $(M=111 \mathrm{~ms}$, $S D=54 \mathrm{~ms}$ ) not being significantly different from lowprobability locations $(M=117 \mathrm{~ms}, S D=54 \mathrm{~ms}), t(14)=$ $1.51, p=.154$. Force of the trigger pull was also not affected across high-probability $(M=0.002)$ and low-probability $(M=$ 0.001 ) locations, $t(14)=1.05, p=.308$.

For the feature (color) probability block, there was no significant effect of probability on RT, with the orientation of high-probability colors $(M=105 \mathrm{~ms}, S D=47 \mathrm{~ms})$ taking as long as the low-probability colors $(M=111 \mathrm{~ms}, S D=52 \mathrm{~ms})$ to detect, $t(14)=1.71, p=.109$. Unsurprisingly, neither IT nor MT nor force was affected by color probability (all $p \mathrm{~s}>.05$ ). The Bayesian tests of color probability on RT in Experiment $1-\operatorname{col}(\mathrm{BF}=9.06[ \pm<0.01 \%])$ and Experiment 2-col $(\mathrm{BF}=$ $0.85[ \pm 0.01 \%])$ clearly support opposing hypotheses. As with Experiment 1, it was again evident that discriminating of orientations (Experiment 2-ori) was in general associated with a larger RT than the discrimination of colors (Experiment 2-col) for the spatial and feature blocks (both $p \mathrm{~s}<.001$ ).

Accuracy and vacillations For Experiment 2-ori, there was an effect of spatial probability on final accuracy, with orientations at the high-probability location being responded to more accurately $(M=94.9 \%, S D=3.3 \%)$ than at the lowprobability location $(M=92.2 \%, S D=6.5 \%), t(14)=2.28$, $p=.039$. There was no effect of spatial probability on the amount of vacillations, with orientations in the highprobability locations being as directly responded to $(M=$ $0.202, S D=0.295)$ as at the low-probability locations $(M=$ $0.206, S D=0.267), t(14)=0.27, p=.790$. Vacillations improved initial to final accuracy $(p \mathrm{~s}<.05)$; this improvement was not significantly different across high $(M=5.7 \%)$ and low $(M=6.2 \%)$ probability locations, $t(14)=.77, p=.453$.

In the feature block, there was no effect of color probability on final orientation accuracy, with orientations of highprobability color being responded to as accurately $(M=$ $92.5 \%, S D=6.6 \%)$ as the low-probability colors $(M=$ $93.8 \%, S D=5.1 \%), t(14)=1.00, p=.336$. As with RT, the Bayesian tests of color probability on orientation accuracy in Experiment 1-col $(\mathrm{BF}=2399[ \pm<0.01 \%])$ and Experiment 2ori (BF $=.402[ \pm 0.01 \%])$ clearly support opposing hypotheses. There was no significant effect of color probability on the amount of vacillations or on the improvement of initial to final accuracy (all $p s>.05$ ). As with Experiment 1, discriminating of orientations (Experiment 2-ori) was overall not significantly more or less accurate than the discrimination of colors (Experiment 2-col), for either the spatial or the feature blocks (both $p \mathrm{~s}>.05$ ).

Postexperiment questionnaires As with Experiment 1, most of the participants did not realize that there were probability manipulations. Of the ones that did (six out of 31 participants), they only described the spatial probability manipulation accurately.

\section{Discussion}

The spatial probability effects from Experiment 1 were replicated. Even though the location was not the dimension that participants had to report, spatial probability effects on RT and accuracy were robust. The effects of spatial probability stem from modulations in IT rather than MT. The same does not hold true for either form of feature probability. When relevant for the task, the effects of orientation or color probability on RT and accuracy are robust (Experiment 1). When irrelevant to the task, their effects are completely eliminated (Experiment 2). This pattern of results is inconsistent with an object-based attention account (e.g. Egly et al., 1994). For example, in Experiment 1, the contingency of location and color has an apparent effect on color detection. If this is because blue objects, being the expected color for that location, are 'attended' to, orientations shown in blue should also be better detected. Instead, Experiment 2 suggests that color probability creates no advantage in orientation detection of colored stimuli.

At first glance, the findings from Experiment 2 seems counter to that demonstrated in the visual search literature. For example, color probability affects search RT even if the response is about orientation (Sha, Remington, \& Jiang, 2017). However, color probability is known to affect search efficiency (Cort \& Anderson, 2013): If the target is likely to possess a certain color, the RT advantage might be due to participants prioritizing the stimuli possessing that color in the search rather than focusing on the stimuli that have nonprobable colors. With the current task, stimuli are only presented one at a time, and therefore color probability is irrelevant to orientation discrimination.

Perhaps a feature-based attention account can explain the differences between Experiments 1 and 2? If orientation probability is irrelevant to color discrimination, there is no need to attend to orientations, explaining the null result in Experiment 2-col. However, this argument cannot apply to Experiment 2ori. The stimuli used are spatial gratings, and while color can be deciphered without having to process orientation information, the reverse is not true: Orientations can be deciphered only by processing (and attending to) the color bands. This idea that orientation perception is more involved than color processing is supported by the finding that orientation 
discrimination consistently took longer than color discrimination. Yet we found that color probability did not affect orientation discrimination on any of our metrics.

While spatial probability creates object-general effects, feature probability creates feature-specific effects. Why does this occur? We have previously (Jabar \& Anderson, 2015, 2017a, b) suggested that orientation probability shares some mechanisms with orientation training effects (such as those that have been demonstrated in monkeys). The tuning functions of orientation selective neurons in V1 that prefer trained orientations are 'sharpened' (Ringach et al., 1997; Schoups et al., 2001). Consistent with these data, orientation probability modulates the electrophysiological $\mathrm{C} 1$ component (reflecting early visual activity) while also modulating the precision of orientation estimations (Jabar et al., 2017). A similar mechanism could also occur for other feature dimensions. Colorselective neurons also exist in V1. The color-tuning of V1 neurons is also narrower than that of its LGN inputs but similar to the downstream IT cortex, suggesting that it has a role in color-processing (Hanazawa, Komatsu, \& Murakami, 2000). There are also subpopulations of color-selective V1 neurons that are selective for orientation (Johnson, Hawken, \& Shapley, 2008). Tuning to probable blue objects would improve the perceptual sensitivity to blue features but might not necessarily improve sensitivity to the orientations of objects presented in blue, depending on which neuronal subpopulation is being tuned by the probability manipulation.

The nonselective benefits of spatial bias can be understood as a gain mechanism (e.g. Carrasco, 2011; Ling et al., 2009). Unlike with orientation probability (Ringach et al., 1997), where only the feature-relevant neurons are facilitated, spatial cues provide no information about the features of the upcoming target stimuli. These are the likely reasons why spatial probability improves detection but not orientation precision (Jabar \& Anderson, 2017b), particularly because it has been argued that tuning changes are required for perceptual precision to change (Yaeli \& Meir, 2010).

If spatial probability affects detection rather than precision (Jabar \& Anderson, 2017b), then an extension of the current task that emphasizes discrimination over detection should further differentiate spatial and feature probability effects. In Experiment 3, a restricted 41-degree range of orientations that bounded a 45-degree orientation was used. The task was to discriminate the more vertical and more horizontal orientations using the same triggers used in the earlier experiments. In addition, this manipulation allowed us to assess perceptual difficulty on a continuous scale by looking at accuracy rates for orientations nearer or further away from the 45-degree boundary. For example, in response to a 44-degree grating, spatial probability might increase the firing rate not just of neurons that prefer a 44-degree stimulus but of all neurons coding for that space. Given that the neurons preferring the 44-degree and 46-degree tilts likely have similar firings to begin with, nonselective gain process should not be expected to help discriminate between the two tilts, because both might be equally gained, resulting in poor performance in this task. In contrast, neural tuning that results from perceptual learning is selective (Ringach et al., 1997; Schoups et al., 2001), and can lead to better perceptual discrimination (Gilbert, 1994; Yaeli \& Meir, 2010). As a result, one might expect that orientation probability improves orientation discrimination even in cases where spatial probability cannot.

\section{Experiment 3}

\section{Method}

Experiment 3 largely followed the design of Experiment 1-ori. Orientation and spatial probability were examined with an orientation discrimination task. The stimuli were shown in the same two locations as in the previous experiments, and in the same two colors. The main difference was that instead of having just two discrete possible orientations ( 45 or 135 degrees), orientations occupied the limited span of 25 to 65 degrees (any integer value, except for the middle 45-degree tilt, was possible). Using the two triggers, participants were asked to discriminate clockwise and anticlockwise from 45 degrees. Orientation probability was manipulated by making either the anticlockwise (25-44 degrees) or clockwise (46-65 degrees) orientations more probable (80\% occurrence). As before, this was counterbalanced by location.

\section{Participants}

Thirty-four additional participants (median age $=19$ years) were recruited (26 females, eight males), in exchange for course credits. Thirty-three reported themselves to be righthanded. The sample size was doubled for this experiment because we wanted to better examine the effect of probability across the different possible orientations. Data from one participant were dropped because of chance-level accuracy (beyond 2.5 SDs of the mean of other participants).

\section{Results}

\section{Reaction time}

In both the spatial and feature probability blocks, RTs and ITs were much longer $(p s<.05)$ than in previous experiments, while MTs were not $(p s>.05)$. The response force was also only about $10 \%$ of those from previous experiments $(p s<.05)$.

There was an effect of spatial probability on RT, with responses to orientations at the high-probability locations being faster ( $M=851 \mathrm{~ms}, S D=136 \mathrm{~ms})$ than at the low-probability location $(M=955 \mathrm{~ms}, S D=140 \mathrm{~ms}), t(32)=9.45, p<.001$. 
ITs were significantly affected by spatial probability, with responses to orientations at the high-probability locations initiated faster $(M=740 \mathrm{~ms}, S D=133 \mathrm{~ms})$ than at the low-probability locations $(M=840 \mathrm{~ms}, S D=149 \mathrm{~ms})$, $t(32)=9.25, p<.001$. However, there was no effect of spatial probability on MTs, with movement times associated with high-probability locations ( $M=110 \mathrm{~ms}, S D=36 \mathrm{~ms}$ ) not being significantly different from low-probability locations $(M=115 \mathrm{~ms}, S D=55 \mathrm{~ms}), t(32)=0.81, p=.422$. Force of the trigger pull was affected, with high-probability locations being responded to more forcefully $(M=0.00018)$ than low-probability locations $(M=0.00016), t(32)=6.63$, $p<.001$.

There was also an effect of feature (orientation) probability on RT, with high-probability orientations being detected faster ( $M=839 \mathrm{~ms}, S D=133 \mathrm{~ms}$ ) than low-probability orientations $(M=881 \mathrm{~ms}, S D=140 \mathrm{~ms}), t(32)=4.04, p<.001$. ITs were significantly affected by orientation probability, with responses to high-probability orientations being initiated faster ( $M=730 \mathrm{~ms}, S D=121 \mathrm{~ms}$ ) than at the low-probability orientations ( $M=767 \mathrm{~ms}, S D=140 \mathrm{~ms}), t(32)=3.77, p<.001$. However, there was no effect of orientation probability on MTs, with movement times associated with high-probability orientations ( $M=110 \mathrm{~ms}, S D=44 \mathrm{~ms})$ not significantly different from low-probability orientations ( $M=114 \mathrm{~ms}, S D=56$ $\mathrm{ms}), t(32)=0.89, p=.379$. Force of the trigger pull was also affected, with high-probability orientations being responded to more forcefully $(M=0.00019)$ than low-probability orientations $(M=0.00017), t(32)=4.09, p<.001$.

\section{Accuracy and vacillations}

In both the spatial and feature probability blocks, accuracy was reduced from previous experiments, as one would predict with a more difficult task $(p s<.05)$. Both vacillations and the improvements from initial to final accuracy were also reduced $(p s<.05)$.

There was a marginal effect of spatial probability on final accuracy, with orientations in the high-probability locations being responded to slightly more accurately $(M=77.6 \%, S D$ $=10.3 \%)$ than in the low-probability locations $(M=75.5 \%$, $S D=12.5 \%), t(32)=1.80, p=.082$. There was no effect of spatial probability on the amount of vacillations $\left(M_{\text {high }}=\right.$ $\left.0.091, S D=0.079 ; M_{\text {low }}=0.093, S D=0.08\right), t(32)=0.38$, $p=.706$. Vacillations improved initial to final accuracy $(p \mathrm{~s}<$ $.05)$, but this improvement was not significantly different across high-probability $(M=1.3 \%)$ and low-probability ( $M$ $=0.8 \%$ ) locations, $t(32)=1.48, p=.148$.

In contrast, there was a significant effect of feature (orientation) probability on final accuracy, high-probability orientations being responded to more accurately $(M=$ $82.0 \%, S D=7.5 \%)$ than the low-probability orientations $(M$ $=72.6 \%, S D=15.2 \%), t(32)=4.89, p<.001$. This accuracy increase was larger than in other experiments $(p \mathrm{~s}<.05)$. There was no effect of orientation probability on the amount of vacillations, with high-probability orientations being as directly responded to $(M=0.096, S D=0.078)$ as the low-probability orientations $(M=0.099, S D=0.088), t(32)=0.38, p=.705$. Vacillations improved initial to final accuracy ( $p$ s $<.05)$, but this improvement was not significantly different across highprobability $(M=0.9 \%)$ and low-probability $(M=1.2 \%)$ orientations, $t(32)=0.81, p=.423$.

\section{Orientation binning}

As a final set of analyses, the different sets of orientations were binned. In the RTs for the feature (orientation) block (see Fig. 5a), there was a significant main effect of probability, $F(1,30)=17.4, M S E=17766, p<.001$, a significant main effect of orientation bin, $F(7,223)=9.25, M S E=21124, p<$ .001 , and no significant interaction effect, $F(7,222)=0.296$, $M S E=13368, p=.955$. On the accuracy scores (see Fig. $5 b$ ) there was a significant main effect of probability, $F(1,32)=$ 26.9, $M S E=0.046, p<.001$, a significant main effect of orientation bin, $F(7,224)=45.32, M S E=0.022, p<.001$, and no significant interaction effect, $F(7,224)=1.22, M S E=$ $0.011, p=.292$.

For the spatial block, there was a significant main effect of probability, $F(1,31)=91.5, M S E=19640, p<.001$, significant main effect of orientation bin, $F(7,223)=$ 17.1, $M S E=17092, p<.001$, and a significant interaction effect, $F(7,222)=2.2, M S E=16079, p=.018$. To qualify this interaction, the maximum effect of bins was looked at for each of the four experimental conditions. This change in RT due to orientation bins was significantly larger for the low-probability locations $(M=250 \mathrm{~ms})$ and opposed to the changes in RT due to orientation bins for the highprobability locations $(120 \mathrm{~ms})$, for low-probability orientations $(140 \mathrm{~ms})$, and for high-probability orientations (130 $\mathrm{ms}$; all $p \mathrm{~s}<.05)$. Changes in RT due to orientation bins among the latter three conditions were not significantly different from each other $(p s>.05)$.

On the accuracy scores while there was a significant main effect of orientation bin, $F(7,224)=51.09, M S E=0.021, p<$ .001 , there was no significant main effect of probability, $F(1$, $32)=2.8, M S E=0.017, p=.103$, and no significant interaction effect, $F(7,224)=1.84, M S E=0.014, p=.080$.

\section{Postexperiment questionnaires}

Again, most of the participants did not realize that there were probability manipulations. Of the ones that did (four out of 33 participants), they only described the spatial probability manipulation accurately. 

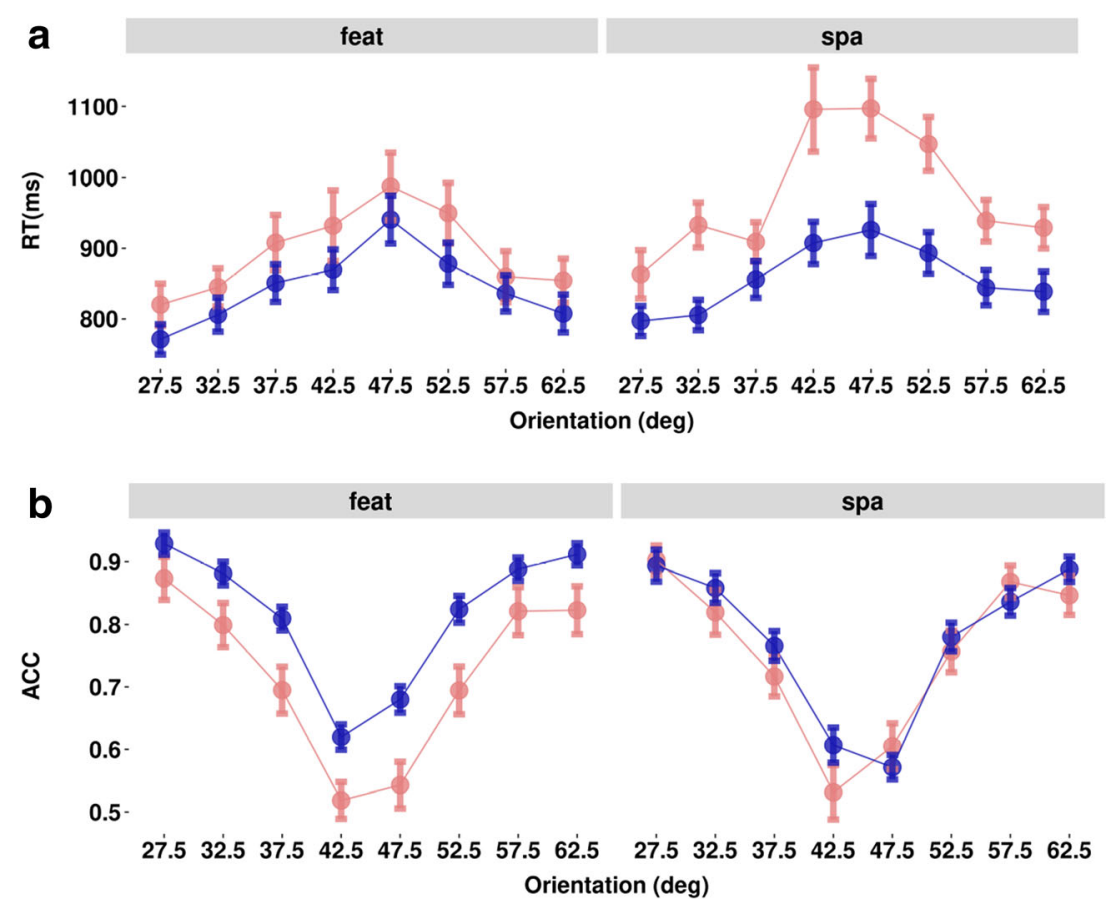

Fig. 5 Reaction time (a) and accuracy across orientations in Experiment 3 (b). Blue (dark) = high-probability; red (light) = low-probability. Error bars represent one standard error. The middle bins correspond to the orientations near the discrimination boundary (45 degrees). (Color figure online)

\section{Discussion}

Experiment 3 was essentially a more difficult version of Experiment 1-ori. Accuracy scores confirmed the increased difficulty. RTs and ITs also increased. The increased difficulty may have also caused participants to be more deliberate because responses were made less forcefully and with fewer vacillations. This is likely what caused differences in response force between high-probability and low-probability locations/ orientations to manifest. Binning the orientations suggests that the difficulty of the discrimination was maximal near the classification boundary, where RTs were longest and accuracy poorest.

As with Experiment 1-ori, RTs were faster for both the probable orientations and the probable locations. However, with the more difficult experiment, the effect on accuracy was wiped out for spatial probability but magnified for orientation probability. Given the hypothesis that only orientation probability should improve perceptual precision when perceptual discrimination is difficult, it would seem puzzling why there would be an interaction between spatial probability and the orientation bins on RT. However, what should be noted is that the accuracy for the orientation discrimination approaches $50 \%$ (i.e. guessing) for the most difficult bin. Additionally, the effect of bins is much larger for the low-probability locations than for the high-probability locations or high/low orientation probability. Together these would suggest that there is a RT cost for guessing at low-probability locations rather than a benefit of perception for high-probability locations. These results match that of Jabar and Anderson (2017b), where orientation probability was found to affect perceptual precision, while spatial probability was only found to affect guessing.

\section{General discussion}

Probable objects are detected better (Hon et al., 2013; Laberge \& Tweedy, 1964; Miller \& Pachella, 1973), as are objects in probable locations (Druker \& Anderson, 2010; Fecteau et al., 2009; Geng \& Behrmann, 2005; Jiang et al., 2015; Rich et al., 2008; Vincent, 2011; Walthew \& Gilchrist, 2006; Wolfe et al., 2007). However, using an orientation-estimation task (Jabar \& Anderson, 2017b), we have previously demonstrated that manipulations of the orientation of a grating leads to effects distinct from spatial probability manipulations. Although both feature and location probabilities shorten reaction time, only feature (orientation) probability increases perceptual precision. Spatial probability affects detection/guess rates, with the precision of estimates of detected objects unchanged.

In this study we provide additional support for the hypothesis that feature and spatial probability effects are distinct, similar to how feature and spatial attention effects are distinct (Carrasco, 2011). Although the RT benefits as a whole seem superficially similar, our method revealed that spatial probability consistently affected only the initiation times. In contrast, feature (orientation/color) probability also modulated the movement times associated with the trigger presses, at least when the detection task was easy (Experiment 1). These data 
highlight that participant 'choices' are often compressions of perceptual and response processes into binary (on/off) button responses. This can create problems when attempting to infer mechanisms from the shape of the RT distributions when a standard keyboard response is used because it would be indecipherable whether the changes in RT derived from changes in perceptual or in response processes.

As a behavioral measure, initiation times might better capture the perceptual component of 'detection' time (Smeets et al., 2016). On the other hand, movement time may more closely track confidence (Jabar \& Anderson, 2015). Shorter movement times suggest that feature probability affects both perception and confidence, the latter of which is diluted when the task is very difficult and confidence generally low (Experiment 3). Increased confidence is not due to prestimulus response preparation, because probable features were spatially contingent, and as spatial locations in the feature block was equiprobable, there was no opportunity to prepare a particular response prior to a stimulus onset. In addition, we found that there were no changes to response preparation prior to the point of response initiation, at which point the initial accuracy and IT already demonstrated effects of spatial and feature probability.

Differences between the probability types extend beyond RT. In Experiment 1, feature probability had a pronounced effect on accuracy. This was amplified when the task was made more perceptually difficult. In Experiment 3, only feature probability affected accuracy. This is likely related to the idea that feature probability improves perceptual precision, while spatial probability does not (Jabar \& Anderson, 2017b). Although spatial probability might help with detection on a coarse level (Experiments 1 and 2), it does not aid in discriminating between similar orientations. This suggests separate mechanisms, particularly as spatial probability effects readily manifest when the response is not dependent on location. Feature probability only seems to manifest when the probable feature is the basis for the perceptual judgement.

What are these mechanisms? Space-based attentional manipulations are thought to invoke gain mechanisms (Carrasco, 2011), such as increasing the input baseline levels of neural activity (Cutrone et al., 2014). Spatial probability has been found to modulate the frontal eye fields and the posterior parietal cortex, a component of the dorsal attention network (Tseng et al., 2013). It could be that because these higher order regions are involved, they modulate the perceptual region in a top-down way, and generate its domain generality.

Feature probability, on the other hand, improves perceptual precision and is relevance specific. In visual search, target color probability has been shown to affect the speed of judging the targets orientation (Sha et al., 2017), presumably by helping in discriminating or prioritizing the target from distractors. However, in our Experiment 2, color probability was not relevant to the orientation of the stimulus, because only the target was on-screen. Not only is this relevancespecificity opposing the trends seen with spatial probability, it is also in contradiction to an object-based attention account (e.g. Egly et al., 1994), because attending to the object with the probable feature should facilitate detection of its other features. An alternative to object-based attention is featurebased attention, which has been suggested to result in neural tuning (Carrasco, 2011; Çukur et al., 2013; David et al., 2008; Ling et al., 2015; Paltoglou \& Neri, 2012), which could explain why perceptual precision is heightened in this (Experiment 3) and previous studies (Jabar \& Anderson, $2015,2017 b)$. It has been suggested that neural tuning is necessary to increase perceptual precision (Yaeli \& Meir, 2010). However, it has also been suggested that feature-based attention may additionally employ a gain mechanism, in addition to neural tuning (Ling et al., 2009). The contributions of topdown and bottom-up processes to tuning changes are presently an open question. Arguing that bottom-up changes may be sufficient are models of orientation training that do not have an 'attentional' component (e.g. Carandini \& Ringach, 1997; Teich \& Qian, 2003), and prior experiments where spatial attention and orientation probability had independent effects (Jabar \& Anderson, 2017a).

Rather than an attentional account, we suggest that our feature probability effects can be parsimoniously explained as experience-dependant neural tuning. This would be similar to how orientation training in monkeys results in the 'sharpening' of the orientation selective neurons in V1 that prefer the trained orientations (Ringach et al., 1997; Schoups et al., 2001). This idea is also consistent with the suggestion that learnt likelihoods are reflected in the early phase of sensory processing (Summerfield \& Egner, 2009), and that the site of plasticity must involve early cortical processing regions with narrow neural tuning (Gilbert, 1994). This neural sharpening argument also explains our recent finding that the electrophysiological $\mathrm{C} 1$ response, related to early $\mathrm{V} 1$ processing ( $\mathrm{Di}$ Russo et al., 2002), is dampened (Jabar et al., 2017), which is unlikely to be explained by a gain mechanism. Coupled with the fact that $\mathrm{P} 1$ is not modulated by orientation probability while it is by feature-based attention (Zhang \& Luck, 2009), feature probability is more likely a low-level perceptual effect than an attentional phenomena.

As an alternative explanation, with feature probabilities in the current task, a participant could be reinforced over time to respond with the probable trigger given the location in which the stimulus was presented. Although it is clear from the data that there was no physical or motor response preparation associated with feature probabilities, if this reinforcement mechanism can speed up decision making without affecting physical response preparation, then it could contribute to the observed feature probability effects. For example, target prevalence has been suggested to affect a decision criterion (Wolfe \& Van Wert, 2010), and perhaps this is true for probabilities 
associated with individual features as well. However, this does not rule out a perceptual locus, because feature probability has also been shown to affect perceptual precision in non-2AFC tasks (e.g. Jabar \& Anderson, 2015, 2017b). It could also be the case that probabilistic information for features might tune relevant perceptual channels, which then affect decision making (Eckstein, Peterson, Pham, \& Droll, 2009). Hinting at this possibility is the finding that both the perception-related $\mathrm{C} 1$ and the decision-related P300 ERP components are modulated by probability (Jabar et al., 2017). The amplitudes of these two components also appeared to be correlated, further suggesting that perceptual and decisional processes are not independent.

Whether feature probability effects occur by reinforcement learning or by perceptual tuning (or both), the key takeaway from this study is that while probability effects are robust and widespread, it is worth noting that they are not all equivalent. Depending on what is made probable, the behavioural effects, and the neural mechanisms involved, could be very different.

\section{References}

Anderson, B. (2014). Probability and the changing shape of response distributions for orientation. Journal of Vision, 14, 15-15.

Carandini, M., \& Ringach, D. L. (1997). Predictions of a recurrent model of orientation selectivity. Vision Research, 37, 3061-3071.

Carrasco, M. (2011). Visual attention: The past 25 years. Vision Research, $51,1484-1525$.

Cort, B., \& Anderson, B. (2013). Conditional probability modulates visual search efficiency. Frontiers in Human Neuroscience, 7. https:// doi.org/10.3389/fnhum.2013.00683

Çukur, T., Nishimoto, S., Huth, A. G., \& Gallant, J. L. (2013). Attention during natural vision warps semantic representation across the human brain. Nature Neuroscience, 16, 763-770.

Cutrone, E. K., Heeger, D. J., \& Carrasco, M. (2014). Attention enhances contrast appearance via increased input baseline of neural responses. Journal of Vision, 14, 16. https://doi.org/10.1167/14.14.16

David, S. V., Hayden, B. Y., Mazer, J. A., \& Gallant, J. L. (2008). Attention to stimulus features shifts spectral tuning of V4 neurons during natural vision. Neuron, 59, 509-521.

Druker, M., \& Anderson, B. (2010). Spatial probability aids visual stimulus discrimination. Frontiers in Human Neuroscience, 4, 1-10. https://doi.org/10.3389/fnhum.2010.00063

Di Russo, F., Martínez, A., Sereno, M. I., Pitzalis, S., \& Hillyard, S. A. (2002). Cortical sources of the early components of the visual evoked potential. Human Brain Mapping, 15, 95-111.

Eckstein, M. P., Peterson, M. F., Pham, B. T., \& Droll, J. A. (2009). Statistical decision theory to relate neurons to behavior in the study of covert visual attention. Vision Research, 49, 1097-1128.

Egly, R., Driver, J., \& Rafal, R. D. (1994). Shifting visual attention between objects and locations: Evidence from normal and parietal lesion subjects. Journal of Experimental Psychology: General, 123,161

Fecteau, J. H., Korjoukov, I., \& Roelfsema, P. R. (2009). Location and color biases have different influences on selective attention. Vision Research, 49, 996-1005.

Filipowicz, A., Anderson, B., \& Danckert, J. (2014). Learning what from where: Effects of spatial regularity on nonspatial sequence learning and updating. The Quarterly Journal of Experimental Psychology, 67, 1447-1456.

Geng, J. J., \& Behrmann, M. (2005). Spatial probability as an attentional cue in visual search. Perception \& Psychophysics, 67, 1252-1268.

Gilbert, C. D. (1994). Early perceptual learning. Proceedings of the National Academy of Sciences, 91, 1195-1197.

Hanazawa, A., Komatsu, H., \& Murakami, I. (2000). Neural selectivity for hue and saturation of colour in the primary visual cortex of the monkey. European Journal of Neuroscience, 12, 1753-1763.

Hon, N., Yap, M. J., \& Jabar, S. B. (2013). The trajectory of the target probability effect. Attention, Perception, \& Psychophysics, 75, 661666.

Jabar, S. B., \& Anderson, B. (2015). Probability shapes perceptual precision: A study in orientation estimation. Journal of Experimental Psychology: Human Perception and Performance, 41, 1666-1679.

Jabar, S. B., \& Anderson, B. (2017a). Orientation probability and spatial exogenous cuing improve perceptual precision and response speed by different mechanisms. Frontiers in Psychology, 8, 183. https:// doi.org/10.3389/fpsyg.2017.00183

Jabar, S. B., \& Anderson, B. (2017b). Not all probabilities are equivalent: Evidence from orientation versus spatial probability learning. Journal of Experimental Psychology: Human Perception and Performance, 43, 853-867.

Jabar, S. B., Filipowicz, A., \& Anderson, B. (2017). Tuned by experience: How orientation probability modulates early perceptual processing. Vision Research, 138, 86-96.

Jiang, Y. V., Sha, L. Z., \& Remington, R. W. (2015). Modulation of spatial attention by goals, statistical learning, and monetary reward. Attention, Perception, \& Psychophysics, 77, 2189-2206.

Johnson, E. N., Hawken, M. J., \& Shapley, R. (2008). The orientation selectivity of color-responsive neurons in macaque V1. The Journal of Neuroscience, 28, 8096-8106.

Laberge, D., \& Tweedy, J. R. (1964). Presentation probability and choice time. Journal of Experimental Psychology, 68, 477-481.

Ling, S., Jehee, J. F., \& Pestilli, F. (2015). A review of the mechanisms by which attentional feedback shapes visual selectivity. Brain Structure and Function, 220, 1237-1250.

Ling, S., Liu, T., \& Carrasco, M. (2009). How spatial and feature-based attention affect the gain and tuning of population responses. Vision Research, 49, 1194-1204.

Mattes, S., Ulrich, R., \& Miller, J. (2002). Response force in RT tasks: Isolating effects of stimulus probability and response probability. Visual Cognition, 9, 477-501.

Miller, J. O., \& Pachella, R. G. (1973). Locus of the stimulus probability effect. Journal of Experimental Psychology, 101, 227-231.

Morey, R. D., Rouder, J. N., \& Jamil, T. (2015). BayesFactor: Computation of Bayes factors for common designs (R Package, computer software). Retrieved from http://cran.r-project.org/ package $=$ BayesFactor

Paltoglou, A. E., \& Neri, P. (2012). Attentional control of sensory tuning in human visual perception. Journal of Neurophysiology, 107, $1260-1274$.

R Core Team. (2016). R: A language and environment for statistical computing. Vienna, Austria: R Foundation for Statistical Computing. URL http://www.R-project.org/

Rich, A. N., Kunar, M. A., Van Wert, M. J., Hidalgo-Sotelo, B., Horowitz, T. S., \& Wolfe, J. M. (2008). Why do we miss rare targets? Exploring the boundaries of the low prevalence effect. Journal of Vision, 8, 11-17.

Ringach, D. L., Hawken, M. J., \& Shapley, R. (1997). Dynamics of orientation tuning in macaque primary visual cortex. Nature, 387 , 281-284.

Schoups, A., Vogels, R., Qian, N., \& Orban, G. (2001). Practising orientation identification improves orientation coding in V1 neurons. Nature, 412, 549-553. 
Sha, L.Z., Remington, R. W., \& Jiang, Y. V. (2017). Short-term and longterm attentional biases to frequently encountered target features. Attention, Perception, \& Psychophysics, 79, 1311-1322.

Smeets, J. B., Wijdenes, L. O., \& Brenner, E. (2016). Reacting with or without Detecting. Motor Control, 20, 200-205.

Summerfield, C., \& Egner, T. (2009). Expectation (and attention) in visual cognition. Trends in Cognitive Sciences, 13, 403-409.

Teich, A. F., \& Qian, N. (2003). Learning and adaptation in a recurrent model of V1 orientation selectivity. Journal of Neurophysiology, 89, 2086-2100.

Tseng, P., Chang, C. F., Chiau, H. Y., Liang, W. K., Liu, C. L., Hsu, T. Y., ... Juan, C. H. (2013). The dorsal attentional system in oculomotor learning of predictive information. Frontiers in Human Neuroscience, 7. https://doi.org/10.3389/fnhum.2013.00404

Vincent, B. (2011). Covert visual search: Prior beliefs are optimally combined with sensory evidence. Journal of Vision, 11, 25. https://doi. org/10.1167/11.13.25
Walthew, C., \& Gilchrist, I. D. (2006). Target location probability effects in visual search: An effect of sequential dependencies. Journal of Experimental Psychology: Human Perception and Performance, 32, 1294-1301.

Wolfe, J. M., Horowitz, T. S., Van Wert, M. J., Kenner, N. M., Place, S. S., \& Kibbi, N. (2007). Low target prevalence is a stubborn source of errors in visual search tasks. Journal of Experimental Psychology: General, 136, 623-638.

Wolfe, J. M., \& Van Wert, M. J. (2010). Varying target prevalence reveals two dissociable decision criteria in visual search. Current Biology, $20,121-124$

Yaeli, S., \& Meir, R. (2010). Error-based analysis of optimal tuning functions explains phenomena observed in sensory neurons. Frontiers in Computational Neuroscience, 4, 130. https://doi.org/10.3389/ fncom.2010.00130

Zhang, W., \& Luck, S. J. (2009). Feature-based attention modulates feedforward visual processing. Nature Neuroscience, 12, 24-25. 